\title{
Structures sociales et blocages techniques dans l'Australie aborigène : quelques éléments critiques
}

\section{Christophe Darmangeat et Jean-Marc Pétillon}

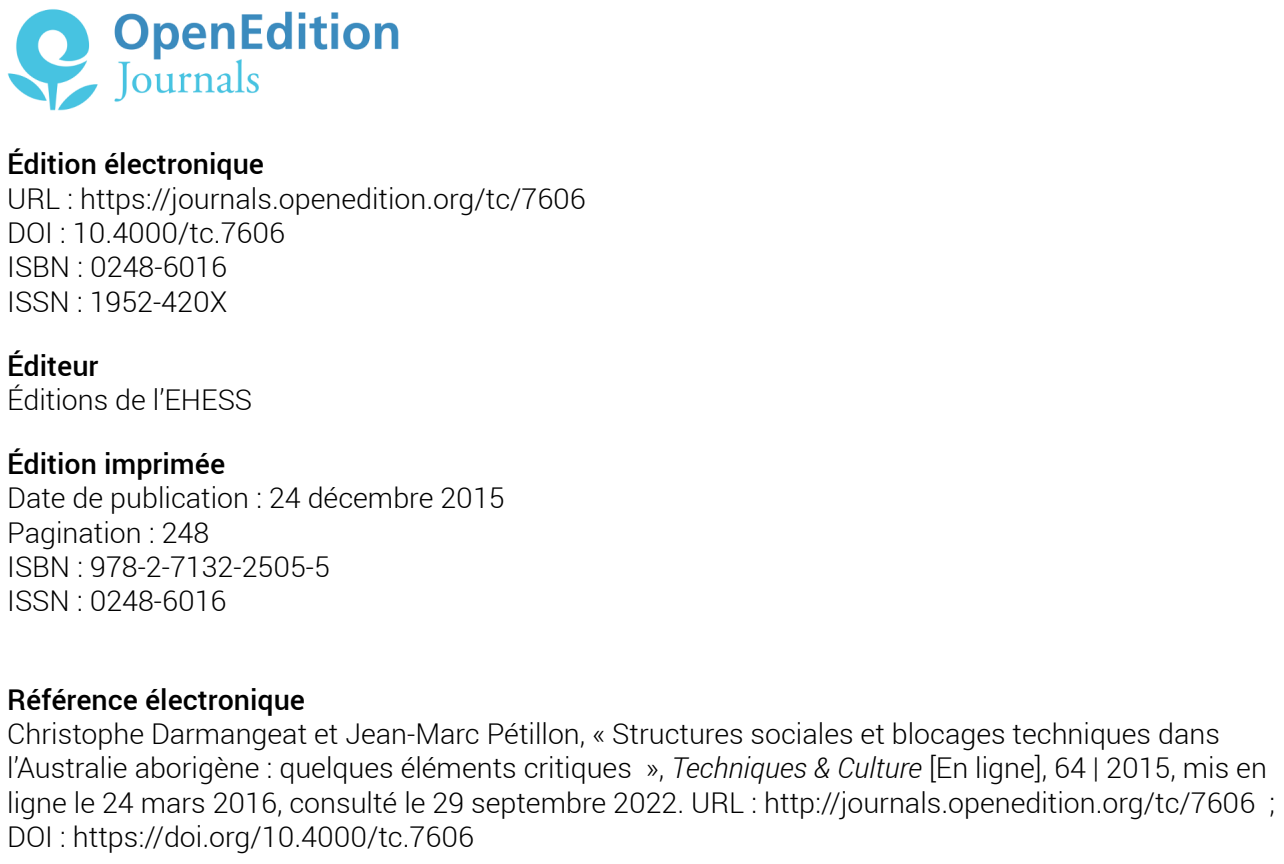

Ce document a été généré automatiquement le 29 septembre 2022.

Tous droits réservés 


\title{
Structures sociales et blocages techniques dans l'Australie aborigène : quelques éléments critiques
}

\author{
Christophe Darmangeat et Jean-Marc Pétillon
}

\section{NOTE DE L'ÉDITEUR}

Nous vous invitons à consulter la synthèse de cet article (édition papier) en téléchargeant le document placé en annexe.

Nous remercions les trois rapporteurs anonymes, dont les remarques ont permis d'améliorer grandement le manuscrit, ainsi que Frédéric Joulian et Lucille Besombes pour leur efficacité éditoriale. Merci également à Marcel Roelandts et Maurice Fhima pour leurs commentaires avisés sur une version antérieure du manuscrit, à Leonn Satterthwait pour ses érudites indications concernant le détroit de Torrès et à Myriam Boudadi-Maligne pour ses informations sur le dingo. Toutes les erreurs restent bien sûr de notre fait. 


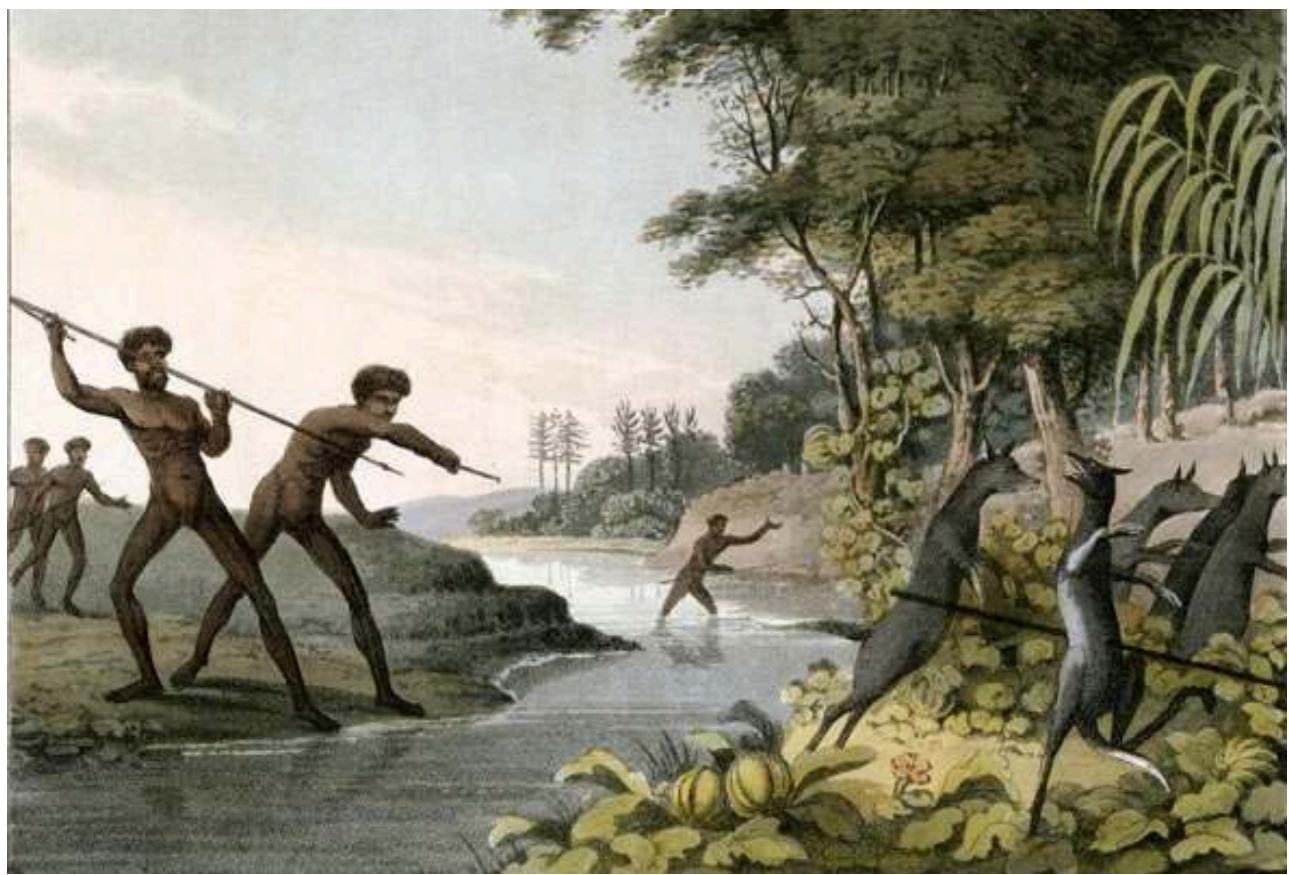

Aquarelle d'Edward Orme (1814)

(c) Field sports of the native inhabitants of New South Wales, Edward Orme (ed.), 1814

La question des rapports entre progrès technique et formes sociales est sans doute l'une des plus difficiles, mais aussi des plus stimulantes, de l'étude des sociétés. En ce qui concerne plus particulièrement les chasseurs-cueilleurs, Alain Testart est un des seuls anthropologues $\mathrm{du} \mathrm{xx}^{\mathrm{e}}$ siècle à avoir formulé une thèse explicite de portée transculturelle. Selon lui, le progrès technique s'est accompli parmi ces peuples à un rythme très inégal, qui ne peut s'expliquer par les seules différences de conditions environnementales: il tient avant tout aux structures sociales, qui peuvent être regroupées en deux grands types exerçant des effets opposés sur l'innovation technique (Testart 1985, 2012). Cette explication est censée éclairer en particulier le retard que présenterait l'Australie aborigène sur les autres régions du globe. A. Testart souligne ainsi que, bien que les tribus australiennes du cap York aient été en contact avec les populations mélanésiennes du détroit de Torrès, plusieurs innovations majeures ne se diffusèrent pas des secondes vers les premières. Il accordait une importance particulière à l'absence, en Australie, de ces quatre éléments que sont l'arc, le chien, le fumage du gibier et l'agriculture (fig. 1). A. Testart renouvelait ainsi l'idée, plusieurs fois avancée, d'un authentique blocage - un « verrou » (Cresswell, 1996: 83) du progrès technique par l'Australie aborigène, blocage qui avait pu être notamment attribué à des valeurs religieuses conservatrices ${ }^{1}$.

1 C'est à l'examen critique de cette thèse que l'article qui suit est consacré. Nous voulons en effet montrer que, dans le cas australien, tout au moins dans les dimensions qui ont trait à la chasse, elle n'est fondée ni empiriquement - dans les données ethnographiques - ni théoriquement. Ainsi, des quatre éléments tenus par A. Testart pour essentiels, seuls ceux qui se rapportent à l'activité cynégétique, à savoir le chien et l'arc, seront discutés. Le fumage du gibier et l'agriculture, qui renvoient aux problématiques du stockage et de la sédentarisation, appelleraient des développements spécifiques qui ne trouveront pas leur place ici. 


\section{Typologie sociale et progrès technique chez les chasseurs-cueilleurs}

Alain Testart occupe une position particulière dans le paysage de l'anthropologie sociale. Évolutionniste déclaré (Testart 1992), il n'en a pas moins rejeté les classifications utilisées par ses homologues américains, critiquant sévèrement la traditionnelle tripartition des sociétés sans États, en bandes / tribus / chefferies (Testart 2005). Outre une érudition et une rigueur de raisonnement rares, A. Testart se distinguait par un intérêt marqué pour les données archéologiques, sans lesquelles tout raisonnement évolutionniste ne pouvait rester à ses yeux que spéculatif. Aussi a-t-il très tôt cherché à relier les analyses sociales qu'il avait pu effectuer à partir de la documentation sur les chasseurs-cueilleurs actuels, ou subactuels, aux séquences archéologiques.

2 L'une des nombreuses originalités de son œuvre est d'avoir accordé une grande importance aux différences de structures sociales exhibées par les différents peuples de chasseurs-cueilleurs, que le néo-évolutionnisme américain a eu coutume de rassembler dans la catégorie indifférenciée de "bandes". Sans même parler des chasseurscueilleurs sédentaires, stockeurs et inégalitaires, tels ceux de la Côte Nord-Ouest (Testart 1982), qui posaient depuis toujours un défi au concept de révolution néolithique, il pensait impératif de différencier deux grands types de sociétés parmi les chasseurs-cueilleurs nomades et économiquement égalitaires.

3 Le premier est celui des Aborigènes australiens. Il se caractérise de prime abord, selon A. Testart, par l'importance des structures de parenté - moitiés, sections et soussections $^{2}$ - qui règlent, en plus des rapports sexuels et des unions matrimoniales, de multiples dimensions des rapports sociaux. A contrario, chez tous les autres chasseurscueilleurs connus, la parenté forme des réseaux beaucoup plus lâches. La plupart de ces peuples, à l'image des Inuits ou des San (ou Bushmen) du sud de l'Afrique, ignorent jusqu'aux clans ${ }^{3}$. Mais, selon A. Testart, la différence la plus essentielle entre les deux types sociaux se trouve ailleurs, dans le type d'obligations liées au mariage. Dans toutes les sociétés sans État, en effet, le mariage implique des prestations, souvent très lourdes, de la part du futur époux à la famille de sa fiancée. Une ligne de fracture décisive sépare les sociétés où le mari fournit un paiement en biens matériels (traditionnellement appelé " prix de la fiancée » dans la littérature anthropologique) de celles où les obligations prennent une autre forme. Les premières sont les sociétés à richesse ; elles sont immanquablement caractérisées par des inégalités matérielles plus ou moins marquées. Les secondes regroupent l'ensemble des chasseurs-cueilleurs nomades, dont les Aborigènes, les San ou les Inuits (sauf ceux des côtes de l'Alaska, stockeurs, sédentaires et qui pratiquaient le prix de la fiancée), chez qui de tels paiements sont inconnus, ce qui fait d'eux des sociétés sans richesse (Testart 2005).

4 Les prestations matrimoniales ne sont pas pour autant absentes chez ces chasseurscueilleurs nomades (auxquels il faut adjoindre les cultivateurs non stockeurs, comme ceux d'Amazonie); elles y prennent même des formes très différentes autour desquelles, selon A. Testart, s'articulent les aspects décisifs de leurs logiques sociales. Dans les sociétés de type $A$ (Australiens), le lien matrimonial contraint le chasseur à fournir, toute sa vie durant, une partie ou la totalité de ses prises à ses beaux-parents ; il s'agit donc d'obligations viagères. Les sociétés de type $B$ (San et autres) regroupent 
plusieurs autres possibilités. Dans l'une d'elles, le futur époux réside avec ses beauxparents et se met à leur service pour un temps souvent indéterminé, mais qui n'excède pas quelques années (l'anthropologie parle à ce propos du "service pour la fiancée »). Ailleurs, les mariages se concluent sous la forme d'échange de sœurs (réelles ou classificatoires ${ }^{4}$, et ne donnent alors pas lieu à une compensation sous forme de prestations.

Qu'il s'agisse des obligations à vie ou du service pour la fiancée, la contrainte pèse lourdement sur les épaules de l'époux. Cependant, leur caractère viager ou temporaire détermine, selon $\mathrm{A}$. Testart, une position très différente du chasseur vis-à-vis de son produit. Dans les sociétés de type $A$, non seulement le chasseur ne conserve rien ou presque rien pour lui-même de son gibier, mais il doit de plus le remettre à des destinataires déterminés; ce transfert résulte donc de la simple exécution d'une obligation et n'engendre aucun prestige social particulier pour celui qui l'accomplit. Dans les sociétés de type $B$, en revanche, une fois le temps d'un éventuel service achevé, le chasseur est propriétaire de plein droit de ses prises. Il en distribuera certes vraisemblablement une partie importante. Mais cette distribution, procédant de sa seule volonté, sera pour lui un moyen de tisser des alliances, de s'attirer reconnaissance et influence sociale.

6 Lorsqu'il se réclamait du marxisme, A. Testart parlait à propos de ces deux formes sociales de "modes de production", un choix qu'il récusa par la suite. Quoi qu'il en soit, ce changement terminologique ne modifiait en rien l'idée selon laquelle ces deux types sociaux déterminaient un intérêt et une attitude très différents face à l'accroissement de la production et au progrès technique :

«S'agissant du chasseur qui distribue lui-même son gibier (ce qui est le cas en type B), il peut trouver intéressant d'adopter une nouvelle technique, plus efficace, parce que cela rendra plus éclatante sa distribution. Mais celui qui n'est pas considéré comme "maître" du gibier ni ne le distribue (ce qui est le cas en type A) n'a aucun intérêt à le faire. Sans doute le beau-frère ou l'ancien qui le fait à sa place y trouverait-il intérêt, mais il n'a pas de moyen de contrôle sur le travail » (Testart 2012 : 274).

7 Cette opposition est reprise et détaillée un peu plus loin :

«Les chasseurs-cueilleurs de type $B$, qui pratiquent normalement le service pour la fiancée, ont de bonnes raisons d'améliorer la production, permettant d'abréger le temps du service ou même de l'abroger en donnant des biens à la place. Rien de tel dans le type $A$, non seulement parce que les obligations sont à vie, c'est-à-dire ne prennent pas fin, mais aussi parce que la quantité de ce qui est demandé n'est pas stipulée comme dans un contrat (qui implique des quantités “déterminées"), étant au gré et à la fantaisie de la belle-mère. C'est pourquoi je dis que le chasseur n'a pas, dans ce type, d'intérêt ni d'incitation à accroître sa production. Quant à ceux qui auraient intérêt à cet accroissement, ils n'ont pas les moyens de le susciter, ce qui a déjà été dit. Mais ils n'y ont pas non plus un intérêt très grand. Sans doute est-ce plus confortable d'avoir de nombreux gendres qui apportent régulièrement du gibier, cela permet de ne pas aller chasser et de s'occuper des choses plus nobles de la religion et du rituel. Mais leur position n'en dépend pas. (...) Sa position ne doit rien à une éventuelle richesse. En bref ni les dominés ni les dominants, d'après ce qu'on voit en Australie aborigène, notre seul exemple actuel de chasseurs-cueilleurs de type A, n'ont de 
véritable raison de développer ni d'améliorer la production matérielle » (Testart $2012:$ 275-276). ignorent la fronde, les bolas, le poison (certes utilisé pour la pêche, mais pas sur les armes de jet), les pièges élaborés (il existait des pièges à poissons, parfois assez monumentaux, mais rien qui utilise des principes mécaniques). Tout cela reste cependant secondaire par rapport aux quatre innovations fondamentales absentes de l'Australie que sont le fumage du gibier, l'agriculture, le chien et l'arc. De l'agriculture et du fumage, on a déjà dit qu'il ne serait pas question dans le cadre limité de cet article ; restent les deux autres.

\section{Le cas du dingo}

En ce qui concerne le chien, on peut s'étonner de ce qu'A. Testart se soit départi dans Avant l'histoire de la prudence qui était la sienne dans le Communisme primitif. À l'époque, reprenant les conclusions de M. Meggitt (1965), il écrivait :

«Il en ressort que l'utilisation du dingo comme chien de chasse était probablement connue en Australie avant l'arrivée des Européens, mais que les conditions du désert expliquent qu'il n'ait pas été utilisé comme tel dans ce milieu» (Testart $1985:$ : 127).

Or, dans Avant l'histoire, le chien australien ne sert plus du tout à la chasse, fût-ce " probablement ", et même en dehors des déserts. Le chien est entièrement devenu ce qu'A. Testart appelle une "invention virtuelle», un élément adopté sans aucune application productive :

«Il est maintenant acquis que (...) le dingo, apporté par les hommes en Australie mais vivant soit à l'état sauvage, soit à l'état domestiqué (ou du moins apprivoisé, parce que les Aborigènes n'en contrôlent pas la reproduction), n'est pratiquement d'aucune utilité ; surtout pas le dingo qui vit parmi les camps, puisque les Aborigènes s'associeront plus volontiers avec un dingo sauvage qu'avec un dingo domestique pour effectuer une chasse à l'issue de laquelle ils laisseront une partie significative aux chiens ; on a pu dire, peut-être par dérision, que le chien ne servait à rien aux Aborigènes, si ce n'est de couverture lors des nuits particulièrement fraîches de cette île grande comme un continent, les hommes s'enroulant contre les feux, avec leur chien derrière eux » (Testart 2012 : 287-288).

Or, cette affirmation soulève au moins deux problèmes. Le premier est que l'opinion de Meggitt, récemment prolongée par Smith et Litchfield (2009), ne fait pas l'unanimité et souffre d'un déficit de sources. Plusieurs auteurs, dont Hayden (1975), ont ainsi relevé des cas où le dingo apprivoisé était utilisé comme un auxiliaire de chasse essentiel. Aux témoignages anciens évoqués par ce chercheur (Nind 1831; Barrington 1802), on 
souhaite ici ajouter celui de James Dawson (par ailleurs souvent cité par A. Testart), qui écrit à propos des Aborigènes de la région de Melbourne :

«Le dingo (...) tient, à juste titre, la première place dans l'estime des Aborigènes. (...) [Les dingos] étaient d'ordinaire élevés à l'état domestique, et on ne se débarrassait jamais des petits. (...) Les chiens étaient entraînés à garder les wuums [campements], ce qu'ils faisaient en grognant et en hurlant. (...) On les dressait également pour la chasse; c'était là leur principal usage. Ils s'employaient habilement à tuer les kangourous, et se faisaient rarement couper par les puissantes griffes postérieures de ces animaux. Lorsqu'ils en tuaient un, ils glapissaient pour avertir leur maître de leur emplacement. Quelques chiens bien entraînés revenaient même au camp pour guider leur maître vers la proie abattue » (Dawson 1881 : 89, souligné par nous).

Pour cette région, le fait est confirmé par William Buckley, observateur privilégié s'il en est puisque, forçat évadé, il vécut plus de trente ans parmi les Aborigènes au tout début de la pénétration occidentale (Morgan 2002 : 56). Un autre Blanc ayant passé plusieurs années isolé dans une tribu, le mousse vendéen Narcisse Pelletier, rapporte à propos du cap York :

« La grande chasse, celle qui demande tout à la fois l'adresse et la ruse, celle que l'on ne peut faire sans l'aide du chien, est la chasse à l'autruche. (...) Nous avons dit que les chiens sauvages sont très communs sur la terre d'Endéavour ; il s'en trouve aussi de parfaitement dressés, et qui pourraient concourir avec nos meilleurs chiens d'arrêt. (...) Quand il part pour la chasse, le sauvage est toujours accompagné de son fidèle Achate »(Merlan 2002 : 98).

Pour le sud-ouest du continent, on lit sous la plume de John Eyre, l'un des meilleurs observateurs du XIX ${ }^{e}$ siècle :

« On tue les chiens sauvages à la lance, mais les petits sont souvent conservés et domestiqués, en vue d'aider à la chasse, pour laquelle ils sont très utiles » (Eyre 1845-II : 284). On pourrait ainsi multiplier les références ${ }^{5}$; loin d'être "virtuelle», l'utilisation cynégétique du dingo par les Australiens était tout à fait tangible, même si elle n'était pas effective au même degré dans tous les environnements et pour tous les gibiers. 


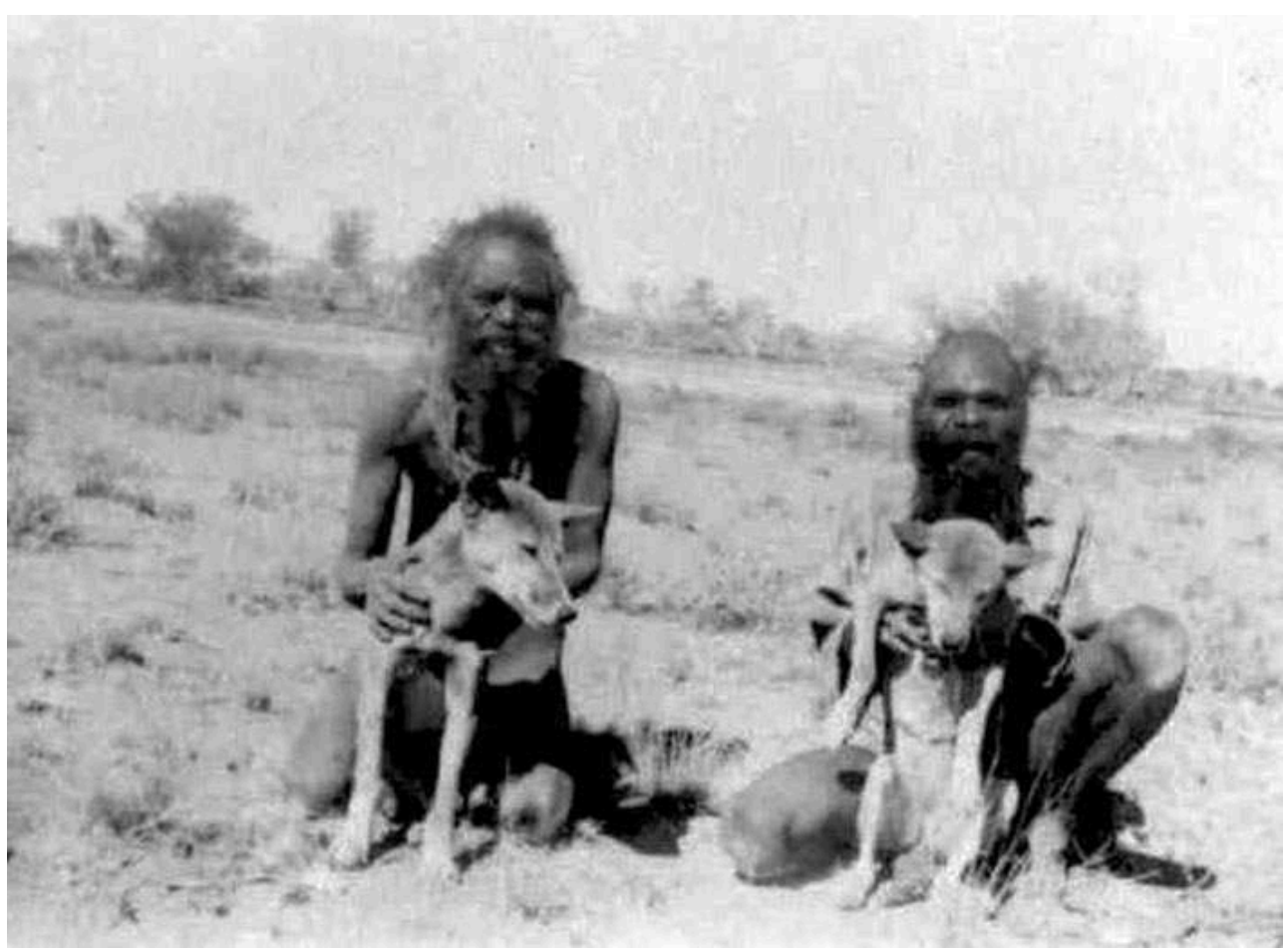

Photographie prise en juin/juillet 1957 dans les Territoires du Nord, lors de l'expédition du lac Mackay (c) Commonwealth of Australia (National Archives of Australia) 2015

Reste le second problème : celui du caractère limité de la domestication du dingo - sur lequel, en revanche, le constat est unanime (fig. 2). Bien qu'apprivoisés depuis leur plus jeune âge (et, pour certains d'entre eux, allaités au sein par les femmes), les dingos s'échappaient fréquemment à l'âge adulte, en particulier lors de la période de reproduction. Cela obligeait les Aborigènes à reconstituer en permanence leur stock par la capture de chiots sauvages.

Commençons par évacuer une question triviale. Si cette amorce de domestication est intervenue plus récemment que dans notre propre Paléolithique (l'époque présumée de la domestication du chien sous nos latitudes étant située a minima au Magdalénien récent, il y a 15000 ans) c'est parce qu'il n'existait pas d'espèce domesticable équivalente en Australie avant que les navigateurs austronésiens n'apportent le dingo, au plus tard il y a 3500 ans (Fillios \& al. 2012). Il n'existe aucun moyen de connaître le délai avec lequel l'animal fut apprivoisé par les Aborigènes; mais on ne saurait attribuer les limites, tout à fait réelles, de cette domestication à un manque de volonté des Aborigènes sans avoir envisagé que ces limites puissent venir du dingo lui-même.

Le degré exact de domestication du dingo au moment de son arrivée sur le continent et sa place dans l'arbre phylogénétique des canidés restent sujets à caution (KolerMatznick 2002 ; Savolainen \& al. 2004). Ce qui est certain, en revanche, c'est que l'état essentiellement sauvage dans lequel il vit depuis des millénaires (sans doute en conjonction avec l'étroitesse de son patrimoine génétique originel) lui a fait développer (ou conserver) des caractères suffisamment originaux pour qu'il soit considéré comme une sous-espèce de Canis lupus - et non comme une simple race. Ainsi, tout comme les louves, les femelles dingo ne sont fécondes qu'une fois par an, contre deux pour les chiens domestiques. 

Occidentaux n'ont pas été longs à remarquer les résistances que cet animal pose à une domestication authentique (Barrington 1798: 75-76), que confirmèrent par la suite de multiples (et toutes infructueuses) tentatives. Un spécialiste a ainsi pu conclure que « Dans toute son histoire, [le dingo] n'a jamais été domestiqué et il ne peut pas l'être » (MacIntosh 1975 : 97).

19 A contrario, on a pu souligner la rapidité avec laquelle les Aborigènes se sont adjoint les services des chiens domestiques importés par les Européens (ou de leur descendance mâtinée avec des dingos). Le cas le plus éloquent est celui de la Tasmanie dont la position insulaire avait empêché le dingo d'arriver jusqu'à elle. Les Tasmaniens (fig. 3) ignoraient ainsi tout de l'existence des canidés, et a fortiori de leur utilité pour la chasse. Mais à partir du moment où les premiers colons occidentaux mirent le pied sur l'île accompagnés de chiens de chasse, il ne fallut qu'une douzaine d'années aux tribus locales pour comprendre le bénéfice qu'elles pouvaient tirer de ce nouvel animal. Elles s'en emparèrent avec avidité (au départ, essentiellement par la force), constituant des meutes fournies qu'elles utilisaient dans leurs activités cynégétiques, et lui ménagèrent une place de choix dans leurs représentations culturelles (Jones 1970).

\section{« Aborigènes de Tasmanie »}

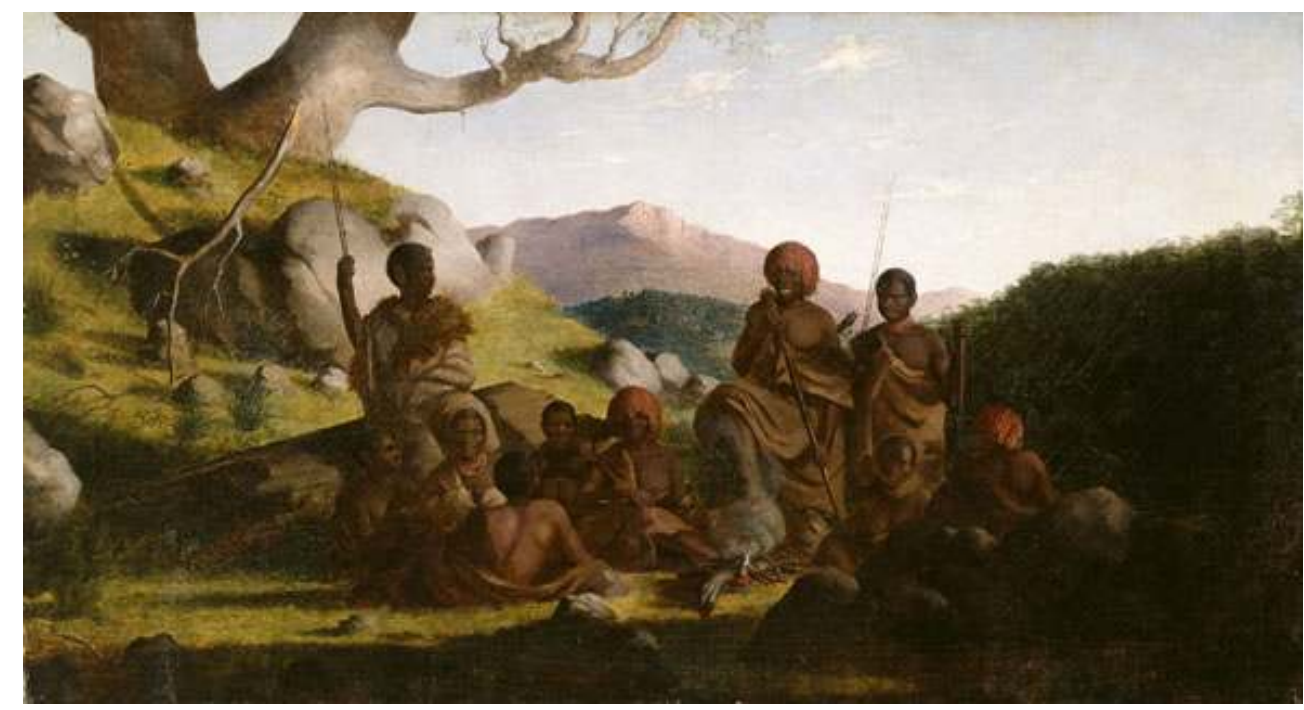

Peinture de Robert Dowling (1856)

National Gallery of Victoria, Melbourne, acquisition 1949 (domaine public)

Tous les indices convergent donc vers l'idée que les Aborigènes ont tiré du dingo tout le parti possible, comme ils l'ont fait ensuite du chien domestique des immigrants européens. En l'occurrence, le seul facteur de blocage qui puisse être mis en évidence est celui qui tient au dingo lui-même.

Il est temps d'en venir à ce «fait majeur » (Testart $2012: 296)$ dont on ne saurait sousestimer l'enjeu :

"L'absence de l'arc en Australie ne revêt toute son importance théorique que lorsqu'on cesse d'y voir une simple curiosité ethnographique pour l'envisager comme l'indice le plus significatif du développement des forces productives propres à la chasse » (Testart $1985: 123)$.

Techniques \& Culture, 64 | 2015 


\section{Arc versus propulseur : un refus du progrès ?}

La question de l'absence de l'arc en Australie se subdivise en deux interrogations. On peut se demander pourquoi les Aborigènes ne l'ont pas eux-mêmes inventé. Mais on peut aussi - et c'est par là que nous commencerons - tenter de comprendre pourquoi ils ne l'ont pas adopté alors que, depuis plusieurs millénaires, les tribus du cap York étaient au contact des Mélanésiens du détroit de Torrès qui, eux, le possédaient ${ }^{6}$. A. Testart rejette avec vigueur les explications environnementales :

«Toute explication écologique est (...) exclue (...), vu la palette très diversifiée des climats qui se succèdent en Australie, du nord tropical au sud tempéré ou atlantique, en passant par les déserts» (Testart 2012 : 296). Il n'est donc qu'une seule explication possible : les Aborigènes ont refusé l'arc pour des raisons sociales, dont on a déjà précisé la teneur. Or, ce raisonnement repose sur au moins deux assertions implicites.

\section{La supériorité de l'arc en questions}

La première est la supériorité absolue de l'arc sur le propulseur. A. Testart sait que ce point a été débattu ; il tient néanmoins le fait que l'arc se soit imposé partout ailleurs dans le monde comme une preuve décisive de sa plus grande efficacité (ibid.). Or, ce raisonnement présente l'inconvénient de se situer à un niveau de généralité très élevé, et ne permet pas forcément de comprendre la manière dont les choses se sont présentées dans des circonstances plus précises - par exemple, celles du cap York.

Il y a quelques années, un bel article de Pierre Cattelain (1994) est venu rappeler que les avantages de l'arc sur le propulseur ne sont pas aussi absolus qu'on a souvent pu le croire. Certes, à peu près partout, sur le très long terme, l'arc a triomphé du propulseur; mais un facteur majeur de ce triomphe est son plus grand potentiel d'améliorations techniques. Si les performances des propulseurs d'aujourd'hui ne dépassent guère celles de leurs homologues australiens, il n'y a pas de comparaison possible entre un arc à poulie moderne et un arc mésolithique. L'arc était susceptible de nombreux progrès, par exemple lorsque son corps est devenu composite ; c'était peutêtre le cas, au moins en partie, de celui qui s'est imposé en Amérique du Nord au cours du premier millénaire de notre ère (Blitz 1988). Mais entre les premiers arcs connus et les propulseurs, la supériorité parait beaucoup plus indécise ; dans certains endroits, le propulseur fut d'ailleurs longtemps conservé conjointement à l'arc. Cela correspondait parfois à un avantage spécifique : celui de pouvoir être manipulé d'une seule main, ce qui permettait d'utiliser l'autre pour manœuvrer une embarcation (comme chez les Inuits) ou pour tenir un bouclier (comme dans certaines troupes toltèques ou maya). En certains lieux, le propulseur avait néanmoins survécu indépendamment de ce bénéfice précis ; un témoin espagnol fait état de son utilisation en 1543 encore par un Indien à l'embouchure du Mississippi (Garcilaso de la Vega 1929 : 354) ; à l'époque historique, il est également attesté en Basse-Californie (Massey 1961), et au XVII siècle, il était l'arme favorite des Tarairu, un peuple amazonien de l'ensemble Tapuya, allié des Hollandais (Prins 2010) (fig. 4). 


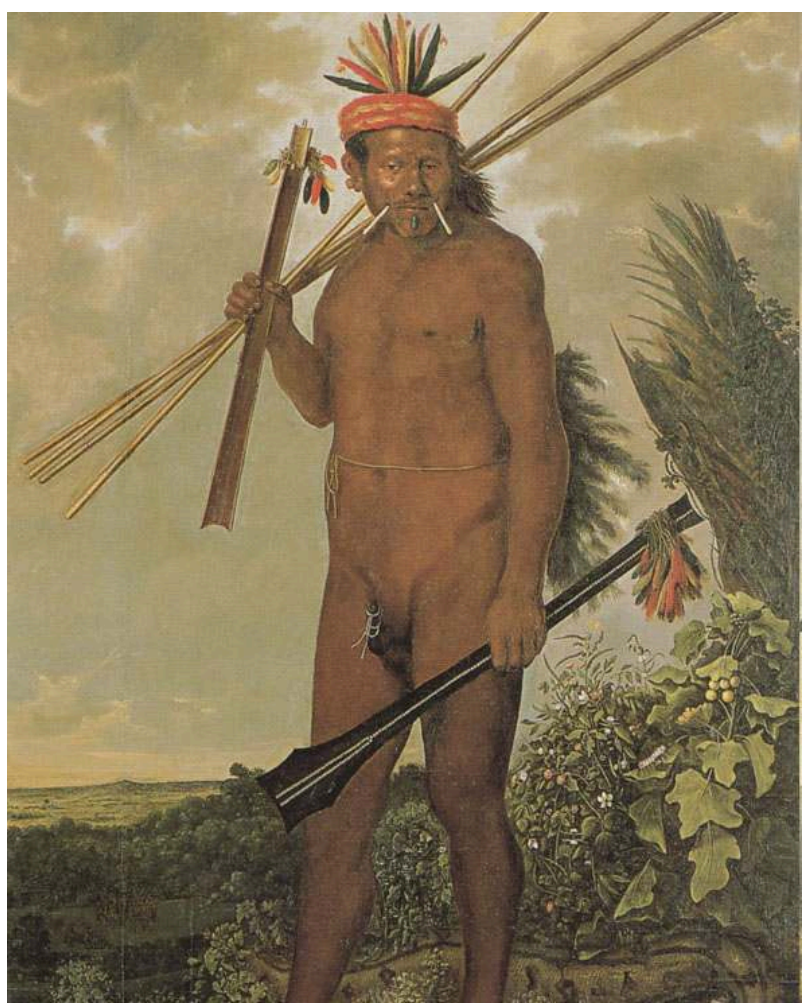

Du peintre hollandais Albert Eckout (1641)

National Museum of Denmark, Copenhague (domaine public)

Si l'arc est généralement considéré comme plus précis et, en tout cas, délivre ses traits à une cadence incomparablement plus rapide que le propulseur, celui-ci utilise en général des projectiles beaucoup plus lourds. Lorsqu'ils font mouche, leur quantité de mouvement supérieure inflige plus volontiers que les flèches des blessures directement incapacitantes. On peut donc penser que si l'arc détient l'avantage sur le propulseur pour des cibles rapides ou de taille réduite, ces avantages sont beaucoup moins clairs sur des cibles plus massives (Tomka 2013).

\section{Ethnographie du détroit de Torrès}

En ce qui concerne le détroit de Torrès, malgré les inévitables lacunes de la documentation, on dispose de suffisamment d'éléments pour ne pas être condamné aux suppositions a priori. C'est d'autant plus nécessaire que la région présente tous les dehors d'un apparent paradoxe. En effet, non seulement l'arc n'a pas pénétré sur le continent pour y remplacer le propulseur ${ }^{7}$, mais c'est le mouvement inverse qui s'est produit : dans les îles de l'ouest du détroit, c'est le propulseur qui a, partiellement ou totalement, supplanté l'arc (fig. 5). 
Carte du détroit de Torrès indiquant la limite de diffusion du propulseur

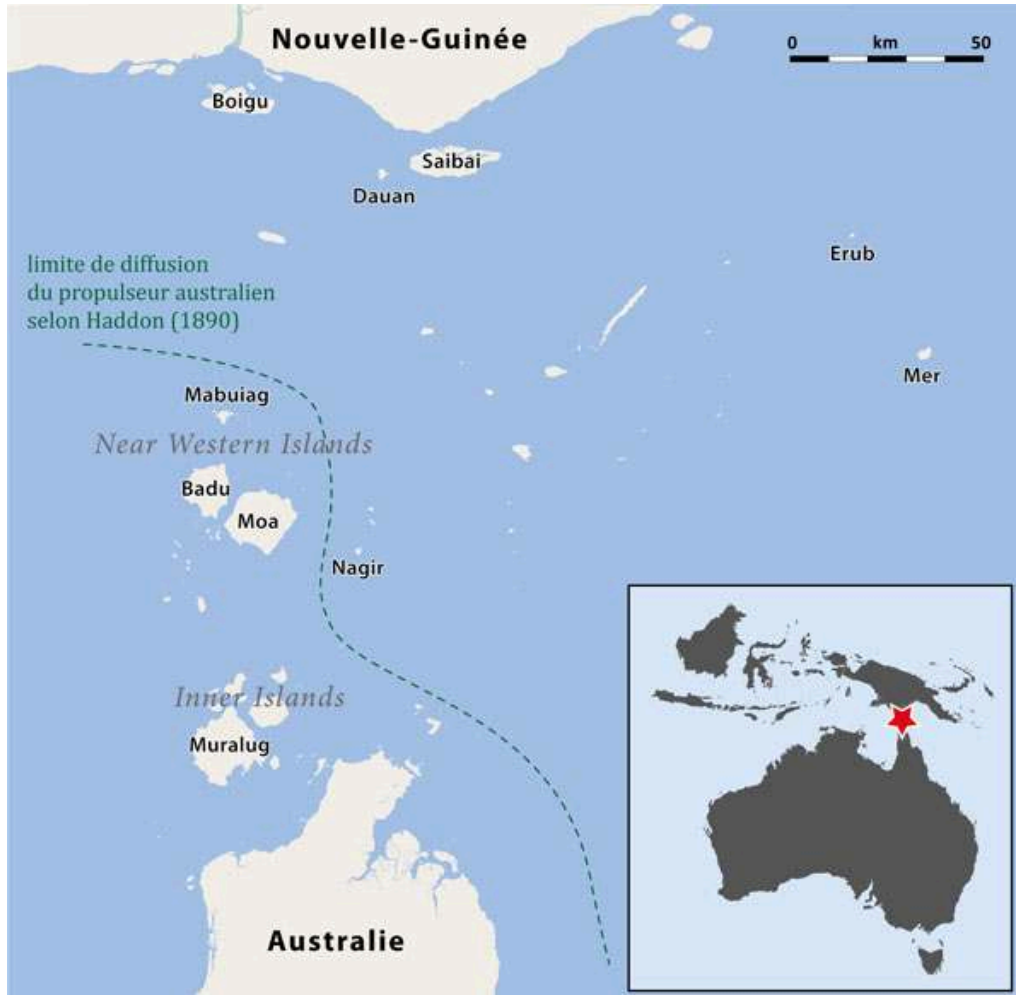

Au sud, le cap York, pointe septentrionale de l'Australie. Au nord, la Nouvelle-Guinée (c) Christophe Darmangeat

A. Testart minimise ce mouvement, écrivant que si le propulseur et ses projectiles étaient devenus des articles d'exportation (quasiment les seuls, au demeurant ${ }^{8}$ ) des Aborigènes vers les Mélanésiens, c'est en raison de la supériorité que les premiers avaient acquise dans cet artisanat (Testart 2012 : 298). Quant aux éventuels avantages $\mathrm{du}$ propulseur sur l'arc, le seul dont les Aborigènes aient fait état était qu'il leur permettait d'avancer dans les hautes herbes en étant mieux dissimulés; il ajoutait ne pas accorder beaucoup de crédit à cette justification (Ibid.).

Les témoignages ethnographiques sur cette zone indiquent cependant que dans certaines de ces îles, les lances australiennes n'avaient pas seulement pris la place des lances locales. Elles y avaient remplacé les arcs, pour la raison la plus simple qui soit: on les considérait comme plus efficaces.

Ce remplacement s'était effectué dans les îles occidentales du détroit, qui se répartissent en deux sous-ensembles distants d'une trentaine de kilomètres. Sur le groupe du nord, celui des Near Western Islands (Moa, Badu, Mabuiag, etc.), situé à michemin entre les côtes papoues et australiennes, la documentation est presque inexistante. Elle est beaucoup plus fournie, en revanche, pour les Inner Islands, plus proches du cap York, en particulier pour son île principale, Muralug (anciennement Prince de Galles). De toutes les îles du détroit, les Inner Islands étaient les seules à ne pas être occupées par une population ethniquement, linguistiquement et culturellement purement mélanésienne. La tribu Kaurareg, dont c'était le territoire, résultait d'une fusion entre les premiers occupants, Aborigènes chasseurs-cueilleurs, et les nouveaux arrivants (Golson 1972). Or, si, sur bien des plans, les Kauraregs avaient conservé les éléments mélanésiens - ils utilisaient notamment des pirogues à balancier 
et cultivaient des végétaux - ils avaient, fait remarquable, presque entièrement abandonné l'arc pour ne conserver que le propulseur.

Le fait est attesté par plusieurs témoignages concordants. Le plus ancien est sans doute celui de James Cook qui, ayant accosté sur l'île Possession (de toutes les Inner Islands, la plus proche du continent), nota que :

" Avant et après avoir jeté l'ancre, nous vîmes quantité de gens sur cette île, armés de la même façon que ceux que nous avions vus précédemment [les Aborigènes, avec leurs propulseurs], à l'exception d'un homme, qui avait un arc et quelques flèches, le premier que nous ayons vu sur cette côte " (Wharton $1893: 311$ ).

Un demi-siècle plus tard, c'est à une observatrice privilégiée, Barbara Thomson, que nous devons nos informations. Naufragée en 1844, à treize ans, elle fut recueillie par les îliens et vécut cinq ans parmi eux avant d'être ramenée à bord du Rattlesnake, où l'artiste Oswald Brierly recueillit son récit :

«Pour la guerre, ils utilisent une lance plus grande appelée ki-kalae, grande lance. Ils n'ont pas de massue. Ils les projettent avec un propulseur, qu'ils appellent kobi. Ils se procurent parfois des arcs depuis les îles plus éloignées. Ils ne s'en servent pas, ils ne les récupèrent que dans le but de les troquer avec les Blancs » (Moore 1979 : 158).

Un autre passager de ce même navire, John McGillivray, rapporte quant à lui :

«Les Kowraregas [Kauraregs] obtiennent les arcs et les flèches de leurs voisins du Nord et s'en servent parfois à la guerre, mais ils préfèrent les lances [et les propulseurs] fabriqués par les Noirs du continent» (McGillivray 1852: 18).

Ces observations seront confirmées et précisées quelques décennies plus tard, en 1890, par Alfred Haddon, membre d'une expédition d'étude dans la région :

«J'ai découvert que l'usage des lances et du propulseur avait été introduit par la tribu occidentale du cap York [les Kauraregs]. Pour autant que je le sache, c'est le seul cas où les Papous ont emprunté quelque chose aux Australiens ; c'était une innovation avisée car, de l'avis général, il s'agit d'une arme plus redoutable. On m'a expliqué qu'il fallait généralement trois ou quatre flèches pour mettre un adversaire hors de combat, tandis qu'une seule lance suffisait d'ordinaire à produire l'effet recherché ; par ailleurs, on vise mieux qu'avec l'arc et les flèches. Toujours à Muralug, j'ai entendu que pour combattre les Blancs, les lances étaient plus efficaces que les flèches. (...) On trouve [les propulseurs] dans les îles les plus occidentales, de Muralug à Mabuiag, mais je ne crois pas que leur usage s'étende au nord jusqu'à Dauan, Saibai et Boigu, ou à l'est jusqu'à Tud et Nagir " (Haddon 1890 : 331-332).

31 En réalité, ainsi que l'indique un témoignage sur l'île de Mer (King 1837: 27), le propulseur australien avait aussi pénétré l'arsenal des habitants de l'est du détroit, sans y acquérir toutefois une place prépondérante : l'arc y restait l'arme principale (fig. 6). 
Des habitants de l'île de Mer (Murray)

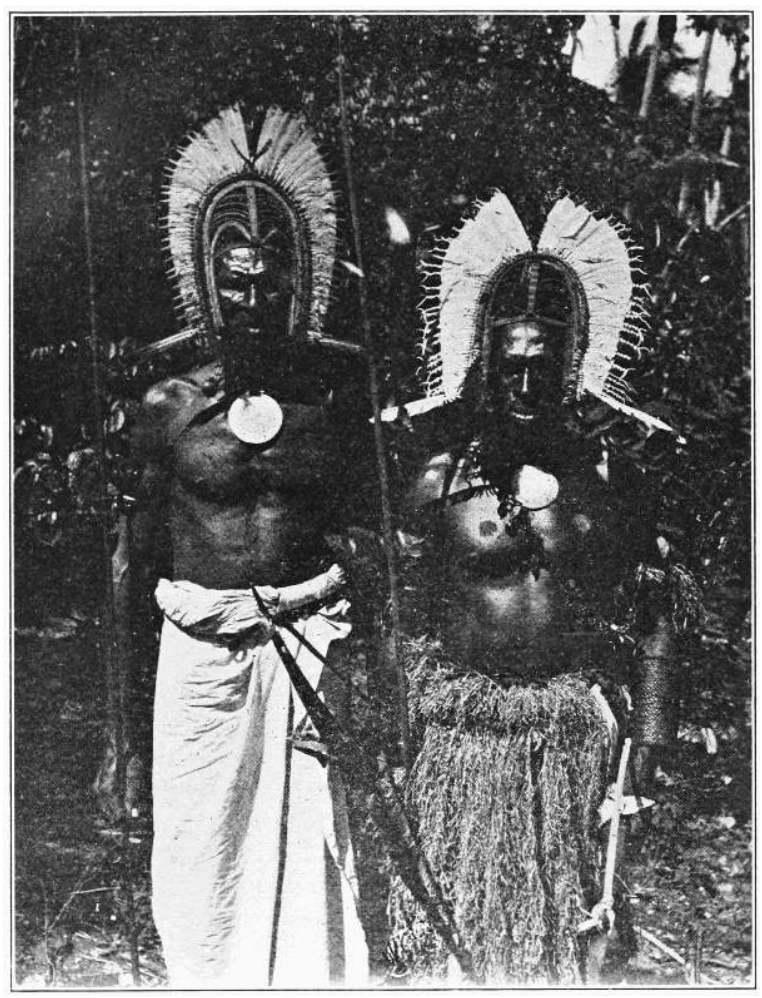

Photographie anonyme, 1914

TIrée de "An expedition to the coral reefs of Torres straits », Alfred Goldsborough Mayer, septembre 1914, p. 226 (domaine public), The Popular Science Monthly Volume 85, juillet-décembre 1914.

Il n'y a aucune raison de douter de la sincérité des motifs donnés par les intéressés pour expliquer leur préférence pour le propulseur. Les Kauraregs vivaient dans un environnement presque dépourvu de proies terrestres. Leurs armes de jet servaient donc pour la pêche et pour la guerre, deux domaines dans lesquels la force des projectiles du propulseur lui permettait de tenir la dragée haute à l'arc. Quant aux tribus aborigènes du continent, leur gibier principal était constitué de wallabies ou de kangourous de bonne taille, pour lesquels, là non plus, l'arc ne possédait sans doute pas d'avantages évidents.

Il faut ajouter à cela que le contact entre Papous et Aborigènes n'a pas pris, comme ce fut sans doute le cas ailleurs dans le monde, la forme d'affrontements dans lesquels les populations dotées de l'arc et de l'agriculture auraient bénéficié d'un net avantage technique et numérique. On ignore si les îles orientales du détroit, exclusivement peuplées de Mélanésiens lors du contact, avaient été le théâtre de telles hostilités. On peut supposer que les arrivants mélanésiens avaient trouvé ces terres vierges. En revanche, dans les Inner Islands, comme on l'a vu, ils avaient rencontré une population d'Aborigènes chasseurs-cueilleurs, avec laquelle ils s'étaient mélangés.

"Ainsi, la confrontation entre les chasseurs-cueilleurs australiens et les horticulteurs papous ne semble pas évidente, même sur le plan économique. (...) De plus, cette confrontation s'est déroulée sur un front extraordinairement étroit, limité au détroit de Torrès lui-même, et dans des circonstances assez exceptionnelles, entre des sociétés de taille réduite séparées par des obstacles maritimes » (Golson 1972 : 390). 

occidentales du détroit de Torrès n'est pas unique. On apprend par un voyageur de la même époque que les habitants de l'île néo-guinéenne de Yule, à plusieurs centaines de kilomètres de là, « préfèrent la lance à l'arc, qui est en train de devenir obsolète parmi eux » (D’Albertis 1880 : 417).

Tous ces éléments plaident en faveur de l'idée que là où les Australiens furent en contact avec l'arc, celui-ci ne présentait pas un avantage décisif sur leurs propres armes traditionnelles, dont ils n'avaient par conséquent pas de raison particulière de se défaire, d'autant que la fabrication d'un arc présente plus de difficultés techniques que celle d'un propulseur (voir par exemple Junkmanns 2001). Le recours aux structures sociales n'est donc pas nécessaire pour expliquer le blocage technique, dans la mesure où celui-ci, en réalité, n'en est pas véritablement un. L'arc n'est pas arrivé au contact de l'Australie comme un progrès qui aurait été rejeté malgré sa supériorité manifeste, mais bien plutôt comme une technologie alternative ne possédant pas, dans les conditions écologiques et politiques de la zone impliquée, d'avantages clairs sur l'armement traditionnel des Aborigènes. Tout au contraire, celui-ci s'est avéré suffisamment convaincant pour que certains des nouveaux venus abandonnent leurs arcs et adoptent le propulseur.

\section{La transition vers l'arc ailleurs dans le monde}

\section{Carte de l'extension chronologique de l'arc en Amérique du Nord}

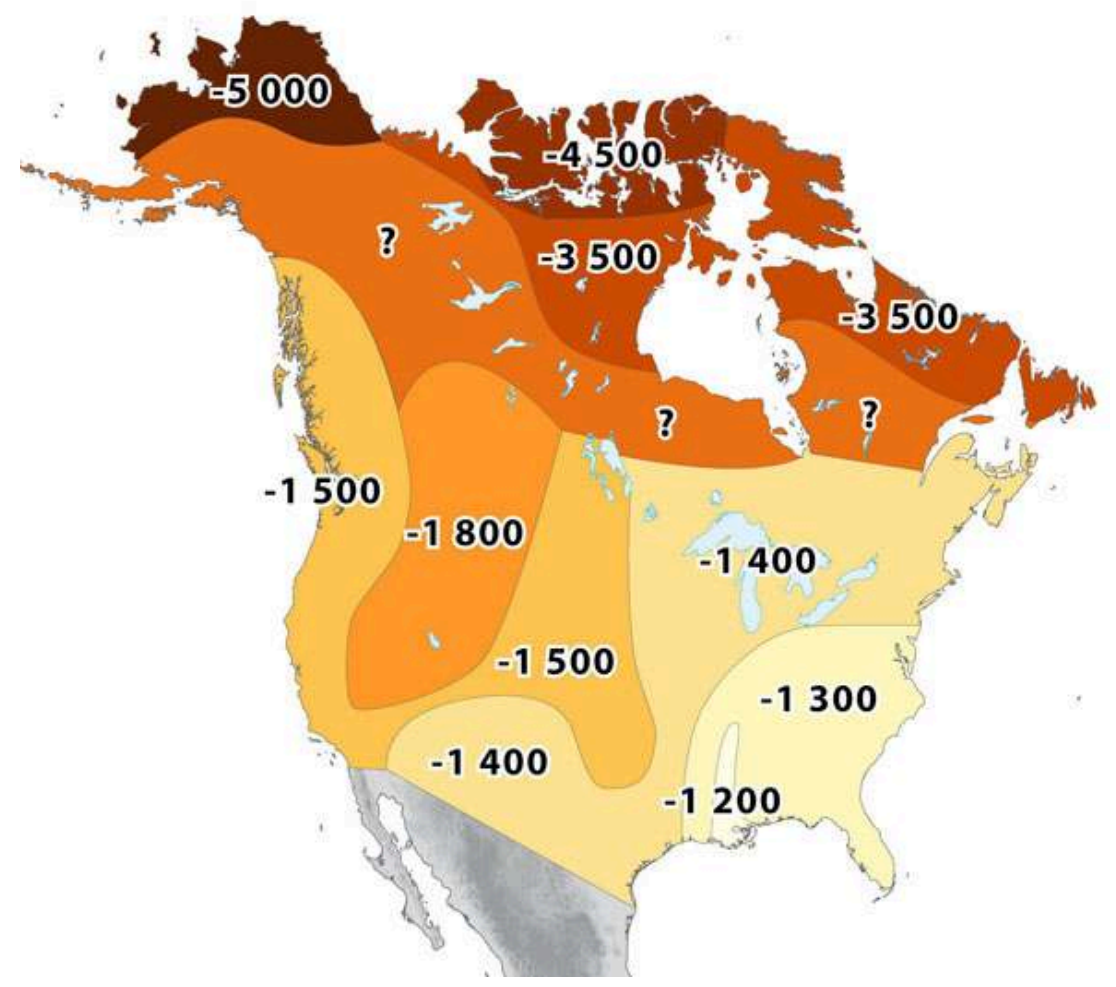

D’après les données de John Blitz (1988). Les dates indiquées sont « avant le présent » (BP)

(C) Christophe Darmangeat

Techniques \& Culture, 64 | 2015 
Sur un plan plus général, ce qui s'est passé dans le détroit de Torrès illustre l'idée plus générale que la supériorité de l'arc ne s'est définitivement affirmée que sur le très long terme, et peut-être seulement après qu'un certain nombre d'améliorations sont intervenues - à la manière dont, des siècles plus tard, l'arme à feu, potentiellement beaucoup plus létale que l'arc, a dû connaître bien des progrès techniques avant de supplanter définitivement celui-ci. On ne peut en effet qu'être frappé par la lenteur de la diffusion de l'arc sur les autres continents, là où l'archéologie permet, malgré toutes les lacunes de nos connaissances ${ }^{9}$, de reconstituer une série temporelle (fig. 7). C'est ainsi qu'arrivé il y a à peu près 5000 ans en Alaska, il a mis près de quatre millénaires pour parvenir jusqu'au Golfe du Mexique. On ne sait pas si A. Testart aurait attribué, là aussi, la lenteur de cette diffusion à la présence de structures sociales de type australien ; il ne s'est semble-t-il exprimé nulle part sur ce point. Mais là encore, point n'est besoin de recourir à cette hypothèse : il suffit de garder à l'esprit que les premiers arcs, s'ils possédaient sans aucun doute certains avantages sur les propulseurs, ne les surpassaient pas, à titre général, de manière indiscutable. Un spécialiste a ainsi pu écrire à propos de l'arrivée de l'arc parmi les cultures andéennes :

« Les mêmes gens utilisèrent des arcs et des propulseurs durant des siècles, et même des millénaires. La technologie de l'arc n'était pas supérieure en tout point et ne se répandit pas parmi les groupes andéens en remplaçant le propulseur dans une vague de progrès technologique révolutionnaire. Au lieu de cela, l'arc fut probablement adopté pour certains usages, tandis que le propulseur continuait à être utilisé pour d'autres. (...) La longue période d'utilisation complémentaire et l'incapacité de l'arc à prouver sa supériorité radicale sur le propulseur suggèrent que l'impact économique et militaire de la technologie de l'arc ait pu ne pas être aussi profond que ce qui a été dit » (Owen 1998: 5).

Les premières traces directes indiscutables de l'usage de l'arc - des hampes de flèches découvertes dans le site allemand de Stellmoor - remontent seulement à la fin du Paléolithique supérieur, il y a 12 000-11 500 ans; les trouvailles d'arcs et de flèches se multiplient ensuite dans les sites mésolithiques d'Europe du nord. La question d'une apparition plus précoce demeure néanmoins posée. Dès le début du Paléolithique supérieur, à plus de 30000 ans avant le présent, les très petites dimensions de certaines pointes de projectile ont conduit plusieurs auteurs à évoquer l'existence, non seulement du propulseur, mais aussi de l'arc (Cattelain 2006). Dans cette hypothèse qui reste très difficile à démontrer - les deux armes auraient cohabité pendant très longtemps, voire se seraient remplacées alternativement selon les époques, avant que l'arc ne l'emporte définitivement, peut-être à la suite de changements environnementaux (tels qu'une généralisation de la forêt tempérée, peu propice à l'usage du propulseur).

Reste un dernier point : la non-invention autochtone de l'arc en Australie. Là encore, il ne paraît pas nécessaire d'avoir recours à l'hypothèse d'une stérilité particulière du milieu social. Sur l'ensemble de la planète, et en insistant une fois encore sur les zones d'ombre qui pèsent sur le raisonnement, l'arc paraît avoir été une invention fort rare. En fait, hormis une origine peut-être européo-asiatique qui a diffusé dans toutes les directions et, via le détroit de Behring, vers l'Amérique du Nord, il n'existe qu'un seul autre foyer présumé de l'arc: en Amérique latine, où il serait apparu il y a 3000 à 1500 ans (Owen op.cit.), avant d'amorcer une lente diffusion. Un simple recours aux probabilités suffit donc à rendre compte des faits connus. Si l'Australie n'a pas inventé 
l'arc, c'est vraisemblablement parce que l'éventualité d'une telle invention, indépendamment de son organisation sociale, était extrêmement faible.

\section{Les structures sociales australiennes}

Les lignes qui précèdent tentent de montrer que les structures sociales australiennes, quand bien même elles auraient possédé les traits que leur attribue A. Testart, ne sont nécessaires pour expliquer ni les limites de l'utilisation du dingo, ni l'absence de l'arc. Ce sont ces structures, ainsi que leurs conséquences supposées sur le progrès technique, que l'on va aborder à présent.

\section{Arme de chasse, arme de guerre}

Contre la thèse selon laquelle le mode de distribution du produit de la chasse serait responsable du blocage du progrès technique dans l'armement, un premier argument vient à l'esprit : dans les sociétés australiennes, tout comme dans celles du détroit de Torrès, les armes ne servaient pas uniquement à chasser, mais aussi à affronter des êtres humains.

Indépendamment de l'épineuse discussion sur le fait que les conflits armés que connaissaient les sociétés aborigènes pouvaient ou non être qualifiés de "guerres ", il est bien établi que ces conflits étaient récurrents. Souvent limités à quelques individus, ils mettaient parfois aux prises des dizaines, si ce n'est des centaines de combattants, occasionnant, en termes relatifs, de nombreuses victimes (Derricourt 1899; Hassall 1902 ; Harvie 1927 ; Warner 1937). On sait également par l'archéologie que de tels affrontements remontent très certainement à une époque reculée (Taçon \& Chippindale 1994).

Or, en admettant même que pour des raisons sociales, les Aborigènes soient restés indifférents à l'efficacité de leur chasse, il est beaucoup plus difficile d'imaginer qu'ils aient pu être pareillement indifférents à l'efficacité martiale de leurs armes remarquons que tous les témoignages, sans exception, précédemment cités à propos de la supériorité du propulseur parlent précisément de son efficacité à la guerre. Si, donc, l'arc s'était avéré une arme d'emblée particulièrement létale, on peine à croire que ces mêmes Aborigènes, qui confectionnaient spécialement des lances barbelées pour infliger à leurs ennemis des blessures plus graves (fig. 8), l'auraient sciemment délaissé. 


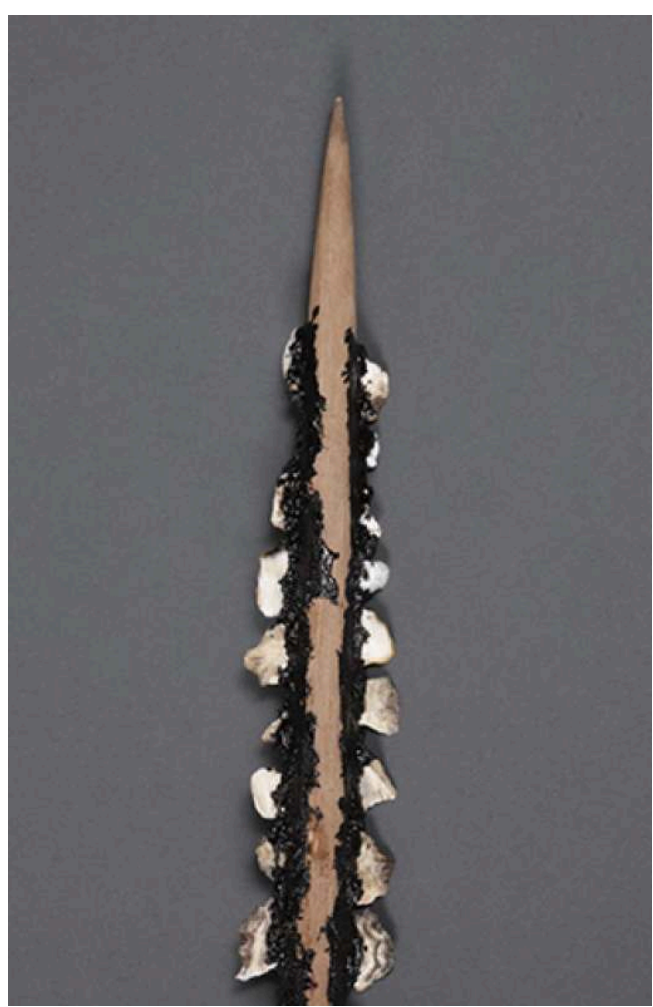

Cette lance, destinée à la guerre, possède des barbillons fabriqués avec des coquilles d'huître, fixés par de la résine. Ces lances étaient particulièrement difficiles à extraire, les barbillons se fracturant et provoquant le décès par infection. Dans l'intérieur des terres, les coquillages étaient remplacés par certains minéraux. Celle montrée ici fut réalisée par l'Aborigène Steven Russell en 2009 pour le musée de Sydney.

http://sydneylivingmuseums.com.au/donate/get-involved/sydneys-story, Musée de Sydney, 2009

\section{Une indifférence à la chasse?}

Concernant la chasse proprement dite, un point frappe d'emblée : au fil des nombreuses pages qu'il a consacrées au désintérêt supposé des sociétés aborigènes pour la chasse et son efficacité, A. Testart n'a jamais fourni une seule illustration ethnographique. Dans l'immense littérature qui traite de ce continent, s'il a pu trouver plusieurs exemples de coutumes contraignant les chasseurs à remettre leurs prises à des tiers, il n'en a jamais produit un seul qui vienne directement appuyer l'idée d'une absence de considération pour cette activité ou pour ceux qui s'y distinguaient. Celle-ci reste donc le fruit d'un pur raisonnement : c'est parce que le chasseur remet l'essentiel de son produit à sa belle-famille, c'est parce que les anciens ne tiennent pas leur position du succès de ceux qui chassent pour eux, qu'il régnerait une absence générale d'intérêt vis-à-vis de la chasse et de ce qui pourrait la rendre plus productive.

Or, les observations ethnographiques viennent unanimement contredire cette déduction. Dans plusieurs tribus, les aptitudes à la chasse étaient un critère essentiel pour juger de la valeur d'un prétendant ${ }^{10}$; et les prestations viagères, loin d'entraîner l'indifférence à la réussite cynégétique, pouvaient au contraire mener à la privilégier dans le choix d'un gendre. A. Howitt rapporte ainsi à propos des Kulin de l'ouest du Victoria : 
« Il n'était pas courant pour un homme d'avoir plus d'une épouse, mais Berak se souvenait d'un individu (...) qui en avait six. En l'occurrence, il s'agissait d'un chasseur réputé, et les hommes lui donnaient leurs filles parce qu'il était capable de leur fournir beaucoup de nourriture. Le devoir d'un homme était de prendre soin de son beau-père, de lui donner des objets (...) et de lui fournir du gibier, qu'on appelait dans ce cas ngul-lurp. » (Howitt 1904 : 256 - souligné par nous). réputation d'un leader ${ }^{11}$. Sur un plan plus général, voici en quels termes est décrit le prestige conféré par cette activité au cap York :

«Une réputation d'habileté à la chasse au dugong et à la tortue était un honneur hautement prisé, et le titre de wotadji, l'homme-dugong (appartenant au dugong) est fort envié. (...) Dans la tribu Koko Ya'o, les hommes âgés disent que si l'on mange de la chair humaine (...) c'est afin de rendre l'homme poi'ya kunta (sans peur) et de lui donner des pouvoirs extraordinaires dans la chasse au dugong » (Thomson $1934: 243$ ). d'outils qui leur auraient assuré de manière évidente un plus grand succès à la chasse (sans reparler de la guerre) ? Ajoutons, pour conclure sur ce point, que l'arrivée de la technique moderne a entraîné les mêmes conséquences que celle du chien domestique : dès qu'ils ont eu accès aux carabines et aux véhicules tout-terrain des Blancs, les Aborigènes n'ont guère montré de réticences à abandonner les propulseurs et la course à pied.

\section{Logiques sociales}

Si, dans le raisonnement d'A. Testart, la conclusion (l'indifférence des Aborigènes au progrès technique) ne se déduit pas des prémisses (le chasseur remet l'essentiel de ses prises à ses beaux-parents) c'est parce qu'on ne peut pas calquer la position sociale du chasseur australien sur celle, par exemple, de l'esclave antique. Même si, dans les deux cas, le producteur n'est pas le propriétaire de son produit, d'autres données interviennent, qui modifient totalement la signification que ce fait peut prendre au sein de la logique sociale d'ensemble.

L'esclave est, à vie, un exploité ; il ne jouit d'aucun droit politique et, le plus souvent, d'aucun droit personnel. Il est méprisé et, avec lui, les tâches qui lui incombent. C'est à cet ensemble de traits que certains auteurs ont proposé d'attribuer l'indifférence, voire l'hostilité supposée des sociétés antiques pour le progrès technique (Finley 1984). Or, la société australienne se situe aux antipodes de cette logique, pour une raison aussi simple que fondamentale: elle ne se divise pas en exploiteurs et exploités. On peut certes y identifier des transferts de biens organisés, essentiellement en direction des 
hommes âgés, dépositaires du savoir religieux et de l'autorité séculière (Darmangeat 2015). Mais outre leur magnitude somme toute limitée, ces transferts ne déterminent pas une coupure entre plusieurs catégories de la population (à tout le moins, de sa fraction masculine) : tout jeune homme a la perspective de devenir un ancien respecté et la dépossession, totale ou partielle, de son produit n'est nullement synonyme d'une dévalorisation de son travail - au contraire, pourrait-on dire : car le même chasseur qui a pour obligation d'approvisionner sa belle-famille est, dans le même temps, lui-même approvisionné par d'autres. Au bout du compte, le système australien assurait une répartition assez égalitaire du produit de la chasse. Ainsi que l'indique l'un des auteurs sur lesquels s'appuie A. Testart :

«Sur le long terme (...) chacun reçoit une part égale de la nourriture collectée, car celui qui donne aujourd'hui recevra demain.» (Mountford $1965: 17)$.

Pour le dire autrement, si les chasseurs australiens étaient dépossédés de leur produit, c'était à titre individuel, mais non à titre collectif. La société australienne n'a par conséquent aucune raison de témoigner, vis-à-vis du travail et de ses résultats, d'un dédain caractéristique de celles où la morale exprime les intérêts et la position d'une classe dirigeante exemptée de toute tâche productive.

Ainsi, même si les coutumes matrimoniales possédaient la généralité et la rigueur que leur prête A. Testart, elles n'entraîneraient pas nécessairement l'indifférence à la production matérielle que celui-ci pensait avoir mise en évidence. Mais il y a plus: même si elle s'appuie sur de nombreuses observations (dont Dawson 1881; Spencer \& Gillen 1899; Mountford 1965 ; Gould 1967; Hamilton 1980 ; Falkenberg \& Falkenberg 1981 ; White 1985), l'idée que, partout en Australie, le chasseur aurait été dépouillé à vie de l'intégralité ou même, d'une fraction importante de ses prises doit sans doute être sérieusement nuancée.

Pour commencer, là où des obligations viagères sont attestées pour tout homme marié (ou engagé dans une promesse de mariage), il faut encore, pour que le prélèvement effectué par la belle-famille soit effectif, que les ayants droit soient présents. Or, la composition des groupes locaux australiens était, comme toujours chez les chasseurscueilleurs nomades, assez fluide. Si lourde que l'obligation ait pesé sur le chasseur, elle pouvait donc ne s'appliquer que par intermittence ${ }^{12}$. En fait, les chercheurs qui se sont intéressés à l'écart entre la rigidité des règles et la souplesse des pratiques ont conclu qu'il était considérable. Chez les Gunwinggu de la terre d'Arnhem, où la distribution du gibier était censée obéir à des normes précises (Altman 1987:133-151), on a ainsi estimé que seule la moitié du gibier était effectivement répartie selon les normes de référence (Peterson 1997 : 183).

Ensuite, on sait de source sûre qu'en certains lieux et pour certains gibiers au moins, les règles pouvaient être très différentes; soit qu'elles n'impliquent pas les parents de l'épouse - dans une tribu du Queensland, une description très précise des prescriptions sur l'attribution des parts du gibier n'implique que les parents du chasseur et aucun de ses affins, sa femme faisant éventuellement exception (Palmer 1884 : 285) - ; soit qu'elle ne s'effectue pas selon des liens de parenté, comme chez les Tiwi de l'île de Bathurst, où les crocodiles étaient partagés entre les trois participants à la chasse : le pagayeur et propriétaire du canot, celui qui transperçait l'animal et celui qui le hissait à bord avait droit à des morceaux déterminés (Goodale 1971 : 170-171). Il en allait de même chez les 
Uutalnganu du cap York pour les poissons pêchés à la lance (Merlan $2002: 103)$; et chez les Murngin de la terre d'Arnhem, la possession d'une arme donnait un droit à une part de tout gibier abattu avec elle (Peterson 1997 : 175).

Enfin, il est permis de douter que les prestations viagères, typiques, par exemple, des Aranda du Désert de l'Ouest, puissent être tenues comme représentatives de l'ensemble du continent australien. De nombreuses occurrences ethnographiques émanant de différentes régions font par exemple état de mariages préférentiels ou subsidiaires par échange de parentes (sœurs réelles ou classificatoires) ${ }^{13}$ - une forme qui, a priori, exclut de telles obligations. En tout état de cause, la diversité et la complexité des prestations matrimoniales aborigènes appelleraient une investigation minutieuse.

Des quatre principaux éléments censés étayer l'idée d'un blocage du progrès technique en raison des structures sociales propres à l'Australie, deux s'avèrent donc peu probants. Tout indique que le dingo a été domestiqué et utilisé dans des activités productives autant qu'il pouvait l'être. Quant à l'arc, s'il a effectivement fait l'objet d'un refus, c'est manifestement parce que dans l'espace limité où il s'est posé comme une alternative au propulseur (celui du détroit de Torrès), il n'apportait aucun bénéfice évident. Ainsi, ni sur le plan empirique, ni, comme on a tenté de le montrer, sur le plan théorique, les structures sociales australiennes ne paraissent constituer un élément d'explication convaincant du niveau technique australien en matière de chasse. (fig. 9) $\mathrm{Au}$ demeurant, il conviendrait également de s'interroger dans quelle mesure l'idée même d'un retard australien dans ce domaine résiste à l'examen. S'ils n'utilisaient ni arcs, ni pièges mécaniques, les Aborigènes n'en avaient pas moins développé divers autres outils d'une indiscutable efficacité ; outre les célèbres boomerangs, on pense en particulier aux filets, d'une variété et d'une taille souvent impressionnantes (Satterthwait 1987). 


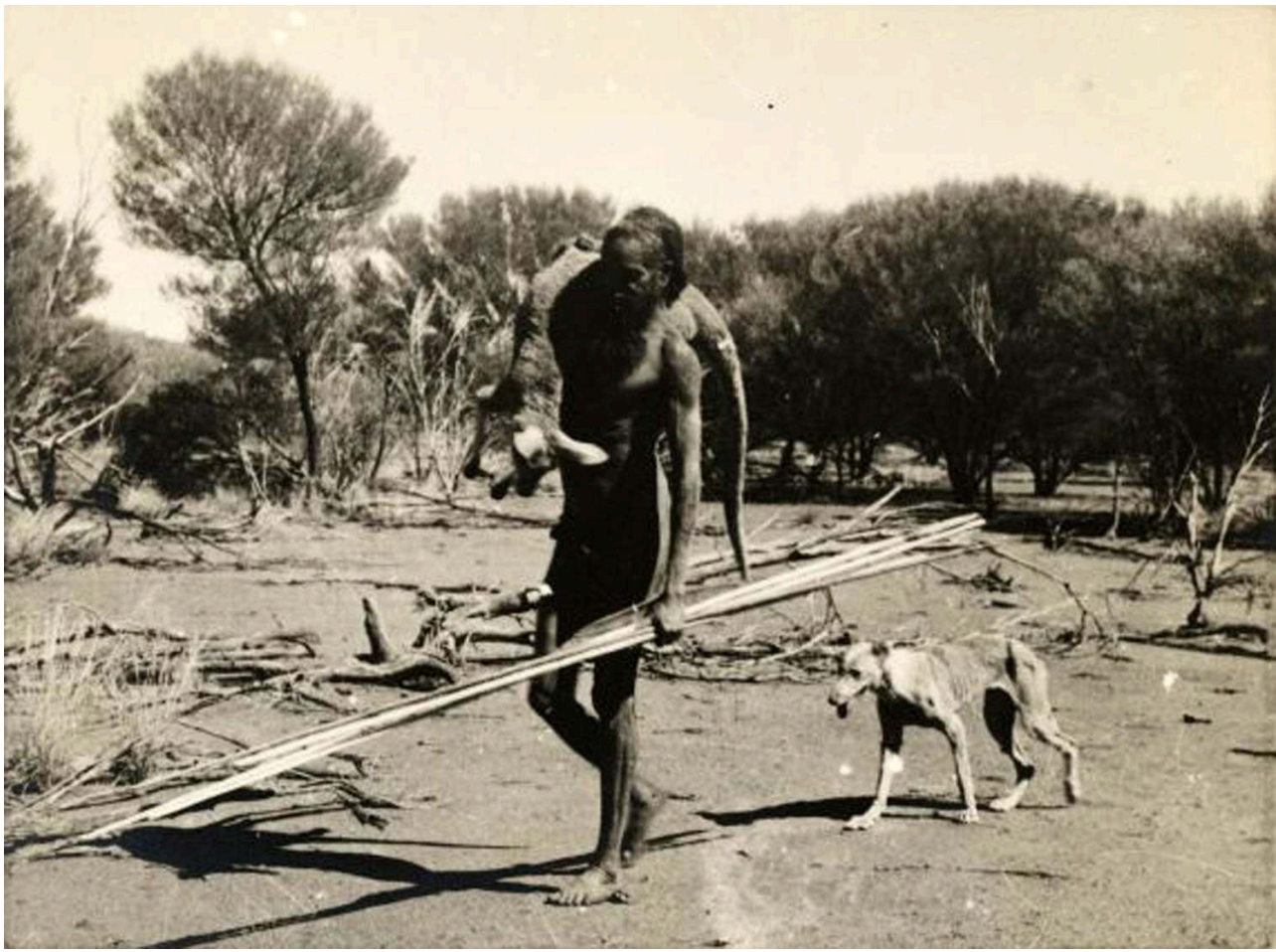

Photographie de Charles P. Mountford (1940), Mont Conner, Northern Territory

Avec l'aimable autorisation de National Library of Australia

Le fumage et l'agriculture, en raison des éléments spécifiques qu'ils soulèvent, n'ayant pu être traités dans le cadre de cette démonstration, celle-ci reste donc nécessairement limitée. Remarquons simplement que la question, au moins en ce qui concerne l'agriculture, a intrigué depuis longtemps et que, face à la thèse sociale, les explications environnementales ont également été soutenues (Golson 1972). Seule une discussion minutieuse sur ce point permettrait de faire éventuellement émerger une conclusion générale sur les rapports entre structures sociales et progrès technique dans l'Australie aborigène, voire au-delà.

\section{BIBLIOGRAPHIE}

Altman, J. C. 1984 « Hunter-Gatherer Subsistence Production in Arnhem Land: The Original Affluence Hypothesis Re-Examined ». Mankind 14 (3) : 179-190.

Altman, J. C. 1987 Hunter-gatherers Today: An Aboriginal Economy in North Australia. Canberra : Australian Institute of Aboriginal Studies.

Barrington, G. 1798 Voyage à Botany Bay. Paris : Desenne.

- 1802 The History of New South Wales, London 
Beveridge, P. 1889 The Aborigines of Victoria and Riverina. Melbourne : M. L. Hutchinson.

Blitz, J. H. 1988 « Adoption of the bow in prehistoric North America », North American Archaeologist $9(2): 123-145$.

Bonwick, J. 1870 Daily Life \& Origins of the Tasmanians. London : Sampson Law, Son \& Marston.

Cattelain, P. 2006 « Apparition et évolution de l'arc et des pointes de flèches dans la Préhistoire européenne (Paléo-, Méso-, Néolithique) », in P. Bellintani \& F. Cavulli dir., Catene operative dell'arco preistorico : incontro di archeologia sperimentale, San Lorenzo in Banale e Fiavè (Trento, Italy) 30.08-01.09.2002, Trento, Soprintendenza per i beni archeologici : 45-66.

- 1994 « La Chasse au paléolithique supérieur : Arc ou propulseur, ou les deux ? ». Archéo-Situla 21-24:5-26.

Clarkson, C. 2016 « Testing archaeological approaches to determining past projectile delivery systems using ethnographic and experimental data », in R. Iovita et K. Sano dir., Multidisciplinary Approaches to the Study of Stone Age Weaponry. Springer (Vertebrate Paleobiology and Paleoanthropology Series).

Cresswell, R. 1996 Prométhée ou Pandore ? Propos de technologie culturelle, Paris : Kimé.

D’Albertis, L. M. 1880 New Guinea : What I Did and What I Saw, t. 1. London : Sampson Low, Marston, Searle \& Rivington.

Darmangeat, C. 2015 « Certains étaient-ils plus égaux que d'autres ? L'exploitation dans les sociétés sans richesse », Actuel Marx 58 : 144-158.

Davidson, D. S., 1934, "Australian Spear-traits and their derivations", The Journal of the Polynesian Society, vol. 43, p. 41-72.

Dawson, J. 1881 Australian Aborigines, George Robertson, Mlbourne, Sydney and Adelaide.

Derricourt, W. 1899 Old Convict Days. London : T. Fisher Unwin.

Eyre, E. J. 1845 Journals of Expeditions of Discovery into Central Australia. t. II London : T. \& W. Boone. Falkenberg, A. \& J. Falkenberg 1981 The Affinal Relationship System. Oslo : Universitetsforlaget.

Fillios, M., Crowther, M. S. \& M. Letnic 2012 « The impact of the dingo on the thylacine in Holocene Australia », World Archaeology 44 (1) : 118-134.

Finley, M. I. 1984 [1953]. Économie et société en Grèce ancienne. Paris : la Découverte.

Foelsche, P. 1886 « Port Darwin : the Larrakia tribe », in E. M. Curr dir., The Australian Race. Melbourne : John Farnes, Government Printer : 250-259.

Gason, S. 1879 " The manners and customs of the Dieyerie tribe of Australian Aborigines ", in J.

D. Woods dir., The Native Tribes of South Australia. Adelaide : E. S. Wigg \& Son : 253-307.

Golson, J. 1972 « Land connections, sea barriers and the relationship of Australian and New Guinean prehistory ", in D. Walker dir., Bridge and Barrier : the Natural and Cultural History of Torres Strait. Canberra : Canberra Research School of Pacific Studies : 375-397.

Goodale, J. 1971 Tiwi Wives, Prospect Heights: Waveland Press.

Gould, R. A. 1967 « Notes on hunting, butchering, and sharing of game among the Ngatatjara and their neighbors in the West Australian Desert », The Kroeber Anthropological Society Papers 36 : 41-66.

Haddon, A. C. 1912 Reports of the Cambridge Ethnological Expedition to Torres Straits, vol. IV, Cambridge University Press. 
Hamilton, A. 1980 « Dual social systems : technology, labour and women's secret rites in the eastern Western Desert of Australia », Oceania 51 (1) : 4-19.

Harvie, W. 1927 « Blacks tribal fight : a unique experience », Coffs Harbour and Dorrigo Advocate, Thursday 14 April 1927.

Hassall, J. 1902 In Old Australia : Records and Reminiscences from 1794. Brisbane : R. S. Hews \& Co.

Hayden, B. 1975 « Dingoes : pets or producers?», Mankind $10: 11-15$.

Holden, R. W. 1879 in G. Taplin, Manners, Customs and Languages of the South Australia Aborigines : 17-28.

Howitt, A. W. 1904 The native tribes of South-East Australia. London : Macmillan.

Hutchings, W. K. 2016 « When is a point a projectile? Morphology, impact fractures, scientific rigor, and the limits of inference », in R. Iovita et K. Sano dir., Multidisciplinary Approaches to the Study of Stone Age Weaponry. Springer (Vertebrate Paleobiology and Paleoanthropology Series). Jones, R. 1970 « Tasmanian Aborigines and dogs », Mankind $7: 256-271$.

Junkmanns, J. 2001 Arc et flèche : fabrication et utilisation au Néolithique. Vienne : Musée Schwab.

King, P. P. 1837 A Voyage to Torres Strait, in Search of the Survivors of the Ship Charles Eaton. Sydney : E. H. Statham.

Koler-Matznick, J. 2002 « The Origin of the dog revisited », Anthrozoös 15 (2) : 98-118.

Krefft, G. 1865 « On the Manners and Customs of the Aborigines of the Lower Murray and Darling ", Transactions of the Philosophical Society of New South Wales, 1862-5 :357-374.

Laade, W. 1973 « Notes on the clans, economy, trade and traditional law of the Murray Islanders, Torres Straits », Journal de la société des océanistes 29 (39) : 151-167.

Lourandos, H. 1997 Continent of Hunter-Gatherers. New Perspectives in Australian Prehistory. Cambridge : Cambridge University Press.

Lumholtz, C. 1889 Among Cannibals. An Account of Four Years' Travel in Australia and of Camplife with the Aborigines of Queensland. London : John Murray.

MacIntosh, N. W. G. 1975. «The Origin of the dingo : an enigma » in M. W. Fox dir., The Wild Canids : Their Systematies, Behavioral Ecology and Evolution. New York : van Nostrand Reinhold : 87-106.

McConnell, U. 1930 « The Wik-Munkan tribe of Cape York Peninsula (II) », Oceania, vol.

$1(2)$

McGillivray, J. 1852 Narrative of the Voyage of HMS Rattlesnake, t. 2, London : T. \& W. Boone.

Massey, W. C. 1961 " The Survival of the dart-thrower on the peninsula of Baja California », Southwestern Journal of Anthropology 17-1: 81-93.

Mathews, R. H. 1905 « Ethnological Notes on the Aboriginal Tribes of Queensland ", Proceedings and Transactions of the Royal Geographical Society of Australasia, Queensland Branch, 20 : 49-75.

McKnight, D. 2005 of Violence, Marriage and Sorcery, Routledge

Meggitt, M. 1964 « Aboriginal food-gatherers of tropical Australia », L'Écologie de l'homme dans le milieu tropical. Morges : IUCN Publications new series 4 : 30-37.

Merlan, C. 2002 [1876] Chez les sauvages : dix-sept ans de la vie d'un mousse vendéen dans une tribu cannibale, 1858-1875. Paris : Cosmopole. 
Meyer, A. 1879 « Manners and customs of the Aborigines of the Encounter Bay Tribe » in J. D. Woods dir., The Native Tribes of South Australia, Adelaide : E. S. Wigg \& Son : 183-206.

Moore, D. R. 1979 Islanders and Aborigines at Cape York. Canberra : Australian Institute of Aboriginal Studies.

Morgan, J. 2002 [1852] The Life and Adventures of William Buckley: Thirty-Two Years a Wanderer Amongst the Aborigines of the then Unexplored Country round Port Philip. Melbourne : Text Publishing.

Mountford, C. 1965 Ayers Rock : Its People, Their Beliefs, and Their Art. Honolulu : East-West Centre Press.

Nind, S. 1831 « Description of the natives of King George's Sound (Swan River colony) and adjoining country », Journal of the Royal Geographical Society of London $1: 21-51$.

Owen, B. 1998 « Bows and spearthrowers in southern Peru and northern Chile : Evidence, dating, and why it matters ", conference paper, third annual meeting of the Society for American Archaeology, Seattle. http://bruceowen.com/research/Owen1998-SAA-

BowsAndSpearthrowersInSouthernPeruAndNorthernChile.pdf consulté le 13 avril 2016.

Palmer, E. 1884. « Notes on some Australian tribes », The Journal of the Anthropological Institute of Great Britain and Ireland 13 : 276-347.

Peterson, N. 1997 « Demand sharing : Sociobiology and the pressure for generosity among foragers » in F. Merlan, J. Morton \& A. Rumsey dir., Scholar and Sceptic, Australian Aboriginal Studies in Honour of L. R. Hiatt. Camberra : Aboriginal Studies Press : 171-190.

Prins, H. E. L. 2010 « The Atlatl as combat weapon in $17^{\text {th }}$-century Amazonia : Tapuya Indian warriors in Dutch colonial Brazil », The Atlatl 23(2) :1-3.

Radcliffe-Brown, A. 1913 « Three Tribes of Western Australia », The Journal of the Royal Anthropological Institute of Great Britain and Ireland $43: 143-194$.

Rots, V. \& H. Plisson 2014 « Projectiles and the abuse of the use-wear method in a search for impact ", Journal of Archaeological Science 48 : 154-165.

Roughsey, D. 1971 Moon and Rainbow. Sydney : Reed.

Satterthwait, L. 1987 « Socioeconomic Implications of Australian Aboriginal Net Hunting », Man, New Series 22-4 : 613-636

Savolainen, P., Leitner, T., Wilton, A. N., Matisoo-Smith, E. \& J. Lundeberg 2004 « A detailed picture of the origin of the Australian dingo, obtained from the study of mitochondrial DNA », Proceedings of the National Academy of Sciences of the United States of America 101 (33) : 12387-12390, doi: 10.1073/pnas.0401814101, consulté le 13 avril 2016.

Schürmann, C. W. 1879 « The Aboriginal tribes of Port-Lincoln : their mode of life, manners, customs, etc. ", in J. D. Woods dir., The Native Tribes of South Australia. Adelaide : E. S. Wigg \& Son : 207-251.

Smith, B. P. \& C. A. Litchfield 2009 « A Review of the relationship between indigenous Australians, Dingoes (Canis dingo) and Domestic Dogs (Canis familiaris) », Anthrozoös 22-2 : 111-128.

Smith, C. 1880 The Booandik Tribe of South Australian Aborigines : A Sketch of their Habits, Customs, Legends and Language, Adelaide : E. Spiller.

Spencer, B. \& F. Gillen 1899 The native Tribes of Central Australia. London : Macmillan.

Taçon, P. \& C. Chippindale 1994 « Australia's ancient warriors : Changing depictions of fighting in the rock art of Arnhem Land, N.T. », Cambridge Archaeological Journal 4 (2) : 211-248. 
Testart, A. 2012 Avant l'histoire. L'évolution des sociétés, de Lascaux à Carnac. Paris : Gallimard.

- 2005 Éléments de classification des sociétés. Paris : Errance.

- 1992 « La question de l'évolutionnisme dans l'anthropologie sociale », Revue française de sociologie 33 : 155-187.

- 1985 Le Communisme primitif(I) : Économie et idéologie. Paris : Maison des Sciences de l'Homme.

- 1982 Les Chasseurs-cueilleurs ou l'origine des inégalités. Paris : Société d'Ethnographie (Université Paris X-Nanterre).

Thomson, D. F. 1934 « The Dugong hunters of Cape York », The Journal of the Royal Anthropological Institute of Great Britain and Ireland $64: 237-263$.

Tomka, S. A. 2013 « The Adoption of the bow and arrow : A model based on experimental performance characteristics ", American Antiquity 78 (3) : 553-569.

Townsend, J. P. 1849 Rambles and Observations in New South Wales. London : Chapman \& Hall.

Vega, G. de la 1829 [1605] La Florida del Inca, t. 7. Madrid : Imprenta de Los Hijos de Dona Catalina Pinuela.

Warner, L. 1937 A Black Civilization. New York ; London : Harper \& Brothers.

Wharton, W. J. L. 1893 Captain Cook's Journal during his first Voyage Round the World 1768-71. London : Elliot Stock.

White, N. 1985 « Sex differences in Australian Aboriginal subsistence : Possible implications for the biology of hunter-gatherers » in J. Ghesquiere, R. D. Martin \& F. Newcombe dir., Human Sexual Dimorphism, London : Taylor and Francis : 323-361.

\section{NOTES}

1. On pense en particulier à Mervyn Meggitt, qui écrit: «Une raison importante pour laquelle [les Aborigènes] ont échoué à élaborer une technique rationnelle de production des végétaux semble avoir été leur philosophie religieuse totémique, qui non seulement fournissait des substituts rituels non techniques à l'action pratique, mais également décourageait, sur le plan moral, l'innovation technique en général. " (1964: 35 ; toutes les traductions des citations en langue anglaise sont de $\mathrm{CD}$ ). Signalons qu'on a parfois, à l'inverse, vu dans certains traits culturels aborigènes un stimulant du progrès technique : ainsi avec Lourandos (1997), pour qui ce sont les vastes cérémonies inter-groupes qui sont à l'origine de l'intensification économique dans le Désert de l'Ouest depuis environ 2000 ans.

2. Ces termes désignent des subdivisions qui découpent respectivement chaque société en deux, quatre et huit fractions, le rattachement d'un individu étant fonction de celui de ses parents selon des règles variables d'un lieu à l'autre.

3. L'anthropologie sociale définit généralement un clan comme un groupe de parenté unilinéaire ; les individus héritent donc de leur rattachement clanique soit par leur père, soit par leur mère.

4. Certains systèmes de parenté appellent «sœur » des parentes qui ne sont pas nécessairement les enfants féminins du père et de la mère du locuteur. Ces systèmes regroupent différentes catégories de parents sous la dénomination de frères, sœurs, pères ou mères, et c'est la raison pour laquelle on les dit « classificatoires".

5. Par exemple, pour la région d'Adélaïde, C. W. Schürmann $(1879: 219)$; Pour le centre, S. Gason (1879, p. 280); pour le Victoria, P. Beveridge (1889: 113); pour la Nouvelle-Galles du Sud, 
J. P. Townsend (1849 : 97) ; pour le Queensland et la péninsule du cap York, U. McConnell (1930 : 201), l'autobiographie de D. Roughsey (1971:53) et le témoignage de C. Lumholtz (1889: 179), dont M. Meggitt ne rend que très imparfaitement compte.

6. À ce fait bien établi, on peut ajouter l'affirmation de Davidson (1934:42) selon laquelle les Européens avaient eux aussi introduit l'arc et les flèches « en tant que jouet dans diverses parties de l'Australie ", mais cette arme "ne semble jamais avoir convaincu les Aborigènes ». Les conclusions que l'on peut tirer de ce passage, que nous n'avons pu recouper avec d'autres informateurs, dépendent beaucoup de la manière dont il convient d'interpréter « en tant que jouet ".

7. La présence de l'arc a été observée au cap York, mais ces armes étaient très probablement entre les mains de visiteurs venus des îles du détroit (Haddon 1912 : 173).

8. Les Aborigènes fournissaient aussi aux îliens la matière première des hampes de flèches, dont ces derniers étaient dépourvus (Laade 1973 : 159).

9. L'arc et le propulseur sont le plus souvent entièrement fabriqués en matières périssables (bois, cuir, tendon, etc.). Leurs traces archéologiques directes sont donc rares et ne reflètent pas forcément la réalité de leur distribution géographique et chronologique dans les sociétés du passé. Faute de telles traces, des indices indirects ont depuis longtemps été recherchés via l'étude des pointes de projectile, qui se conservent mieux (elles sont souvent en pierre ou en matières osseuses). On tente de distinguer les pointes de sagaie des pointes de flèche, soit par leurs dimensions (les pointes des projectiles tirés au propulseur sont en moyenne plus grandes et plus lourdes que celles des flèches tirées à l'arc), soit par les traces d'impact qu'elles portent (en moyenne encore, les tirs de sagaie infligent aux pointes des dégâts plus importants que les tirs à l'arc). Ces méthodes restent controversées, notamment, dans les deux cas, à cause de l'amplitude du recouvrement entre les deux catégories. Le débat dure depuis des décennies sans qu'une position de consensus ne se dégage (pour une revue critique récente, voir Rots \& Plisson 2014 ; Clarkson 2016 ; Hutchings 2016).

10. En Tasmanie : «Un bon chasseur, capable de remplir tant l'estomac de beau-papa que celui de sa fille, était un parti estimé » (Bonwick 1870: 61). Dans la région de Melbourne : «Les seules cérémonies préparatoires au mariage qu'ils connaissent consistent en premier lieu à obtenir le consentement des parents, le meilleur argument du prétendant étant d'être un bon guerrier et un chasseur expert - de sorte d'être capable de protéger et de nourrir sa famille » (Morgan 2002 : 70). Au Nord, dans le Golfe de Carpentarie : «Un mauvais chasseur avait peu de chances d'attirer les femmes ou d'être vu d'un œil favorable par ses belles-mères et beaux-pères potentiels » (McKnight $2005: 26$ ).

11. "Si le chef est un homme capable, montrant du courage au combat et de l'habileté à la chasse, les autres chefs lui offrent souvent des femmes" (Dawson 1881: 35). "Si [le leader] possède une autorité largement reconnue, ce qui est incontestablement le cas de certains d'entre eux, c'est parce qu'il se trouve à la tête d'un groupe numériquement important, qu'il est luimême célèbre pour son habileté à la chasse ou au combat ou pour sa connaissance des anciennes traditions et coutumes de la tribu. » (Foelsche $1886: 11$ )

12. Ainsi chez les Murinbata de la terre d'Arnhem : «Normalement, la personne à qui ego fournit le tartar [la prestation viagère] ne vit pas dans sa horde, et le tartar n'est donc pas fourni de manière quotidienne. Mais d'un autre côté, les membres des différentes hordes et tribus sont ensemble si souvent qu'un homme passera une partie importante de son temps avec le père, la mère, l'oncle maternel et le frère de sa femme, et en ces occasions, les obligations de l'homme s'exercent en priorité vers ces personnes. » (Falkenberg 1981 : 45). Cet extrait met l'accent sur les périodes où les prestations issues du mariage sont fournies, mais on peut à tout aussi bon droit insister sur celles où elles ne le sont pas. 
13. Par exemple : A. Radcliffe-Brown (1913 : 156), R. H. Mathews (1905 : 54), C. Lumholtz (1889: 164), A. Howitt (1904: 236-237, 244, 262, 263-264), C. Smith (1880), G. Krefft (1865 : 372), R. W. Holden $(1879$ : 28$)$, H. E. A. Meyer (1879 : 190).

\section{RÉSUMÉS}

Selon A. Testart, il faut distinguer, parmi les chasseurs-cueilleurs nomades et économiquement égalitaires, deux grands types de sociétés. Le premier (type A), dont les Aborigènes australiens sont le seul exemple dans le présent ethnographique, se caractérise par des structures sociales décourageant l'innovation technique; il s'oppose en cela au second type (B) où ce blocage technique n'existe pas. A. Testart expliquait ainsi le « retard technique » de l'Australie aborigène, en particulier l'absence de ces quatre éléments clés que sont le fumage du gibier, l'agriculture, le chien et l'arc. Cet article est consacré à l'examen critique de cette thèse, en se concentrant sur les éléments ayant directement trait à la chasse : le chien et l'arc. Nous montrons que le dingo domestique, loin d'être dépourvu d'applications productives, est un auxiliaire de chasse essentiel pour de nombreux groupes australiens; et que ses caractéristiques naturelles suffisent à rendre compte de l'inachèvement de sa domestication. Quant à l'arc, en particulier dans ses versions les plus primitives, les avantages qu'il offre par rapport au propulseur doivent être relativisés; en témoignent, dans l'histoire des techniques, la lenteur de sa diffusion et sa cohabitation fréquente avec le propulseur. Et de fait, l'ethnographie du détroit de Torrès nous montre que, lorsque les Aborigènes du cap York - utilisateurs de propulseur - entrèrent en contact avec les Mélanésiens - utilisateurs d'arc -, c'est le propulseur qui supplanta l'arc auprès de plusieurs populations mélanésiennes, parce qu'on le considérait comme plus efficace. Les structures sociales australiennes ne sont donc nécessaires pour expliquer ni les limites de l'utilisation du dingo, ni l'absence de l'arc. De surcroît, plusieurs éléments laissent penser que ces structures n'avaient en réalité aucune raison d'entraîner une indifférence particulière pour le progrès technique, en particulier dans le domaine de la chasse.

According to A. Testart, two types of societies must be distinguished among nomadic, economically egalitarian hunter-gatherers. The first type (A), the only example of which, in the ethnographic present, is the Australian Aboriginals, is characterized by social structures that discourage technical innovations. Conversely, in the second type (B) this technical block does not exist. This is how A. Testart explained the "technical backwardness" of Aboriginal Australia, especially the absence of four key elements : game smoking, agriculture, the dog, and the bow. This paper is a critical review of Testart's theory centered on the two elements most directly linked to hunting: the dog and the bow. We show that the domestic dingo, far from being deprived of productive usage, is actually an essential hunting aid for many Australian groups ; and that the incomplete domestication of the dingo is due to its intrinsic characteristics as a species rather than to a lack of domestication effort from the Aboriginals. As for the bow, the advantages of the primitive bow over the spearthrower must be strongly qualified. In the history of techniques, this lack of decisive advantage is evidenced by the slow diffusion of the bow and its frequent co-occurrence with the spearthrower. And the ethnography of the Torres Strait shows that, when the Cape York Aboriginals (using the spearthrower) met the Melanesians (using the bow), it is the spearthrower that replaced the bow among several Melanesian groups, simply because it was considered more efficient. The Australian social structures are therefore not 
necessary to explain either the limited use of the dingo or the absence of the bow. Furthermore, ethnographic evidence suggests that there is actually no reason to think that these social structures imply a particular indifference for technical progress, especially in hunting techniques.

INDEX

Keywords : Alain Testart, Australia, bow, dingo, domestication, spearthrower, technical evolution

Mots-clés : Alain Testart, arc, Australie, dingo, domestication, évolution technique, propulseur

\section{AUTEURS \\ CHRISTOPHE DARMANGEAT}

Université Paris-Diderot

JEAN-MARC PÉTILLON

Université de Toulouse, CNRS, laboratoire TRACES 